\title{
HOLOMORPHIC MOTIONS AND QUASICIRCLES
}

\author{
GAVEN J. MARTIN
}

(Communicated by Mario Bonk)

\begin{abstract}
We give a new application of the theory of holomorphic motions to the study of the distortion of holomorphic maps of disks and annuli establishing sharp distortion theorems.
\end{abstract}

The theory of holomorphic motions, introduced by Mané-Sad-Sullivan [8, has had a significant impact on the theory of quasiconformal mappings. A reasonably thorough account of this is given in our book [1. In [9] we established some classical distortion theorems for quasiconformal mappings and used the theory to develop connections between Schottky's theorem and Teichmüller's theorem. Here we further explore these ideas to give new distortion theorems. A sequel discusses further interesting applications to the theory of mappings of finite distortion and their boundary values. The results here also give new estimates for the distortion of extensions of analytic germs as studied in [5].

\section{Definitions}

We first recall the two basic notions we will need for this paper.

1.1. Quasiconformal mappings. A homeomorphism $f: \Omega \rightarrow \mathbb{C}$ defined on a domain $\Omega \subset \mathbb{C}$ and in the Sobolev class $f \in W_{l o c}^{1,2}(\Omega, \mathbb{C})$ of functions with locally square integrable first derivatives is said to be quasiconformal if there is a $1 \leq K<$ $\infty$ so that $f$ satisfies the distortion inequality

$$
|D f(z)|^{2} \leq K J(z, f), \quad \text { almost everywhere in } \Omega .
$$

Here $D f(z)$ is the Jacobian matrix and $J(z, f)$ its determinant. The basic theory of quasiconformal mappings is described in [1].

1.2. Holomorphic motions. The theorem quoted below, known as the extended $\lambda$-lemma and first proved by Slodkowski [13, is key. The dilatation estimate was observed by Bers and Royden earlier. See 4 for a discussion. A complete and accessible proof can be found in [1, Chapter 12]. First we give the definition of a holomorphic motion.

Let $X \subset \hat{\mathbb{C}}=\mathbb{C} \cup\{\infty\}$ be a set. A holomorphic motion of $X$ is a map $\Phi$ : $\mathbb{D} \times X \rightarrow \hat{\mathbb{C}}$ such that:

- For any fixed $a \in X$, the map $\lambda \mapsto \Phi(\lambda, a)$ is holomorphic.

Received by the editors September 27, 2011 and, in revised form, January 23, 2012.

1991 Mathematics Subject Classification. Primary 30C62, 37F30, 30 C75.

This research was supported by the Marsden Fund. The author thanks Aalto University and the University of Helsinki, where this research was initiated. 
- For any fixed $\lambda \in \mathbb{D}$, the map $a \mapsto \Phi(\lambda, a)$ is an injection.

- $\Phi(0, a)=a$ for all $a \in X$.

Note especially that there is no assumption regarding the measurability of $X$ or the continuity of $\Phi$ as a function of $a \in X$ or the two variables $(\lambda, a) \in \mathbb{D} \times X$.

Theorem 1. Let $\Phi: \mathbb{D} \times X \rightarrow \hat{\mathbb{C}}$ be a holomorphic motion of $X$. Then $\Phi$ has an extension to $\hat{\Phi}: \mathbb{D} \times \hat{\mathbb{C}}$ which is a holomorphic motion of $\hat{\mathbb{C}}$, and for each $\lambda \in \mathbb{D}$

$$
\hat{\Phi}_{\lambda}=\hat{\Phi}(\lambda, \cdot): \hat{\mathbb{C}} \rightarrow \hat{\mathbb{C}} \quad \text { is } \frac{1+|\lambda|}{1-|\lambda|} \text {-quasiconformal. }
$$

Moreover, if $\rho_{\mathbb{D}}$ denotes the hyperbolic metric (curvature $=-1$ ) of the unit disk, then for $\lambda_{1}, \lambda_{2} \in \mathbb{D}$ the map $\hat{\Phi}_{\lambda_{1}}^{-1} \circ \hat{\Phi}_{\lambda_{2}}$ is $K$-quasiconformal, with $\log K=\rho_{\mathbb{D}}\left(\lambda_{1}, \lambda_{2}\right)$.

\section{Holomorphic MOTIONS OF CIRCLES}

A $K$-quasicircle $\Gamma$ is a Jordan curve in the plane which is the image of the unit circle under a quasiconformal map of $\hat{\mathbb{C}}$. If a Jordan curve is a quasicircle, then it is often possible to get an upper and lower bound on the number $K$; however, getting sharp estimates is extremely rare. The place to start is to consider ellipses. We give the following lemma, which must be well known but which we could not explicitly find in the literature. Our proof follows from interesting work of S. Krushkal [6] and R. Kühnau [7] on extremal quasiconformal reflections.

Lemma 1. For $0<r \leq 1$ the ellipse $E_{r}=\left\{e^{i \theta}+r^{2} e^{-i \theta}: \theta \in[0,2 \pi]\right\}$ is a $K$ quasicircle, where $K=\sqrt{\frac{1+r^{2}}{1-r^{2}}}$. This estimate is best possible.

Proof. $E_{r}$ is the image of the unit circle under the map $J_{r}(z)=z+r^{2} / z,|z|>1$, with affine extension $\tilde{J}_{r}(z)=z+r^{2} \bar{z},|z| \leq 1$. The extremal dilatation among all quasiconformal reflections across the ellipse $E_{r}$ is known to be $Q_{r}=\left(1+r^{2}\right) /$ $\left(1-r^{2}\right)$, and the map $J_{r}$ has Grunsky norm $\kappa\left(J_{r}\right)=k\left(J_{r}\right)=r^{2}$, where $k\left(J_{r}\right)$ is the Teichmüller norm of $J_{r}$, defined to be the minimum of dilatations $k(w)=$ $\|\bar{\partial} w / \partial w\|_{\infty}<1$ of all quasiconformal extensions of $J_{r}$ across $S^{1}$ (preserving orientation). These facts can be found in the survey [6]

On the other hand, due to Ahlfors-Kuhnau, for any quasicircle $\mathcal{C}$ its reflection dilatation $Q_{\mathcal{C}}$ is related to its extremal dilatation $K_{\mathcal{C}}$ of quasiconformal maps $f$ : $\hat{\mathbb{C}} \rightarrow \hat{\mathbb{C}}$ of the extended complex plane with $f(\mathbb{S})=\mathcal{C}$ by $Q_{v}=K_{\mathcal{C}}^{2}$. So $K_{E_{r}}=\sqrt{Q_{r}}$. This can be found in the survey [7]. The result follows.

Note that the extremal mapping constructed above is not linear (unless it is the identity). However, the Beltrami coefficient of this extremal mapping (we have not proved uniqueness) has constant modulus, $|\mu|=r^{2}$.

Let $\Phi: \mathbb{D} \times \mathbb{D}$ be a holomorphic motion of the disk and let $\hat{\Phi}: \mathbb{D} \times \hat{\mathbb{C}}$ be the extension given by Theorem 1 . According to Theorem 1 the set $\Phi_{\lambda}(\mathbb{S})=\hat{\Phi}_{\lambda}(\mathbb{S})$ is a $\frac{1+|\lambda|}{1-|\lambda|}$-quasicircle. However, this estimate ([1, Theorem 12.5.1]) can be improved by symmetrisation of the Beltrami coefficient as in [1, §13.3.1 and Theorem 13.3.5]. This leads directly to the following theorem.

Theorem 2. Let $\varphi: \mathbb{D} \rightarrow \mathbb{C}$ be a conformal mapping. Then for all $0<r<1$, $\varphi(\mathbb{S}(r))$ is a $\sqrt{\frac{1+r}{1-r}}$-quasicircle. 
The first part of the proof is to consider the motion defined by $(\lambda, z) \mapsto \frac{1}{\lambda} \varphi(\lambda z)$ once $\varphi$ is normalised so that $\varphi(0)=0$ and $\varphi^{\prime}(0)=1$. The extended $\lambda$-lemma extends $\varphi \mid \mathbb{D}(0, r)$ to a $\frac{1+r}{1-r}$-quasiconformal map of $\mathbb{C}$ which the symmetrisation across $\mathbb{S}(r)$ reduces to a $\sqrt{(1+r) /(1-r)}$-quasiconformal map $f$ of $\mathbb{C}$ with $f(\mathbb{S}(r))=$ $\varphi(\mathbb{S}(r))$. Using the Möbius group of the disk we obtain the following corollary which we will use later.

Corollary 1. Let $\varphi: \mathbb{D} \rightarrow \mathbb{C}$ be a conformal mapping and $D=\mathbb{D}(a, r) \subset \mathbb{D}$. Then $\varphi(D)$ is a $K_{a, r}$-quasidisk, where

$$
K_{a, r} \leq\left(\frac{(1+r)^{2}-|a|^{2}}{(1-r)^{2}-|a|^{2}}\right)^{1 / 4}
$$

Moreover, $\varphi \mid \mathbb{D}(a, r)$ has a $K_{a, r}^{2}-$ quasiconformal extension to $\mathbb{C}$.

2.1. Sharpness and examples. The nice thing about Theorem 2 is that it provides explicit distortion bounds on the constants associated with a quasidisk. Following from these are a vast number of consequences for the function theory of the domain. For instance, quasidisks are uniform domains and the distortion estimates bound the constants of uniformity. However, it is reasonable to ask about sharpness. The obvious candidate to consider here is the Koebe function $z \mapsto \frac{z}{1+z^{2}}$. After inversion, application of Corollary 1 implies that if $|a|+r<1$, then

$$
\mathcal{C}_{a, r}=\left\{a+r e^{i \theta}+\frac{1}{a+r e^{i \theta}}: 0 \leq \theta \leq 2 \pi\right\}
$$

is a $K_{a, r}$-quasicircle. With the choice $a=0 \in \mathbb{D}$, Corollary 1 implies that the ellipse $E_{r}=\left\{e^{i \theta}+r^{2} e^{-i \theta}: 0 \leq \theta \leq 2 \pi\right\}$ is a $\sqrt{(1+r) /(1-r)}$-quasicircle. We already know the best possible estimate here is $\sqrt{\left(1+r^{2}\right)\left(1-r^{2}\right)}$.

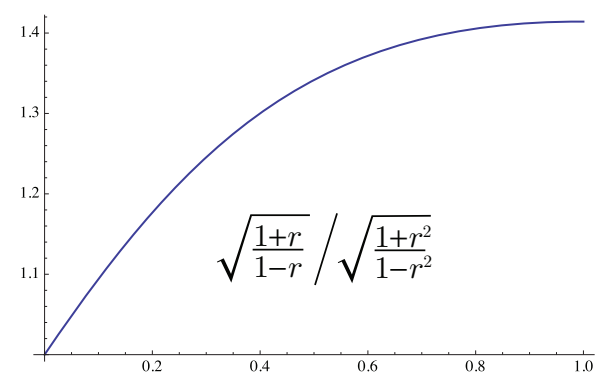

This graph shows that the estimates of Corollary 1 are not too bad. They are off by at most a factor of $\sqrt{2}$. Below we illustrate two families of $\sqrt{2}$-quasicircles obtained from Corollary 1 and the map $z \mapsto z+1 / z$ on various disks of hyperbolic radius 2 . 

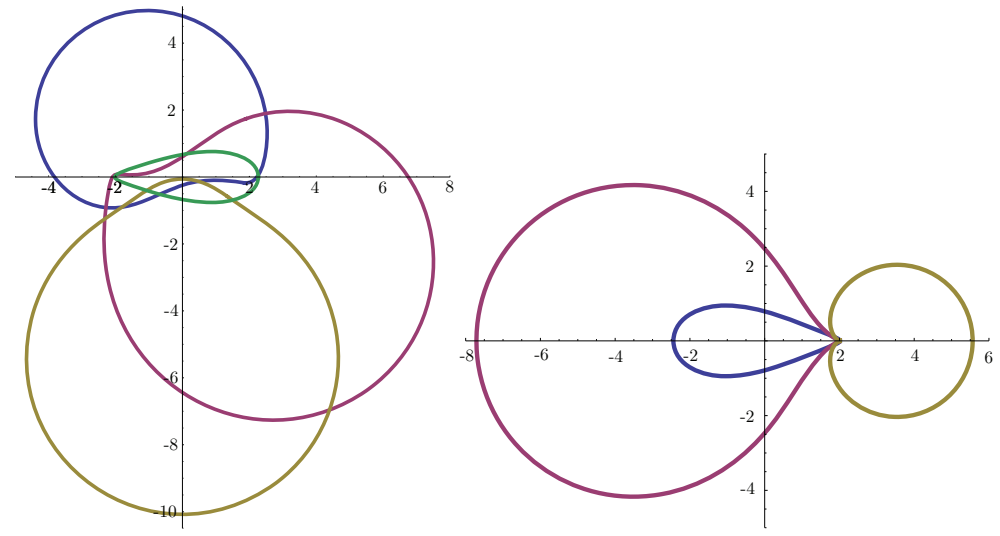

3. AnNuli

Next we want to consider the case of conformal mappings of annuli. There are two natural questions. We could consider a conformal map $\varphi$ defined on the annulus $\mathbb{A}(r, 1 / r)=\{z: r<|z|<1 / r\}$ and ask for bounds on the distortion of the quasicircle $\varphi(\mathbb{S})$ in terms of $r$. Here we first study the distortion of the circles $\varphi(\mathbb{S}(r))$ when $\varphi$ is a conformal map of $\mathbb{A}\left(r_{0}, 1\right)$ with $\varphi(\mathbb{S})=\mathbb{S}$. This is because of an interesting application in obtaining distortion bounds for the boundary values of mappings of finite distortion; see [10. These maps arise when one "integrates" a compactly supported Beltrami coefficient to a self-mapping of finite distortion of the disk.

Suppose that $\varphi: \mathbb{A}\left(r_{0}, 1\right) \rightarrow \mathbb{D}$ is a conformal mapping such that $\lim _{|z| \rightarrow 1}|\varphi(z)|=$ 1. The Carathéodory theorem and the Schwarz reflection principle imply that $\varphi$ extends by reflection to a conformal mapping $\hat{\varphi}: A\left(r_{0}, 1 / r_{0}\right) \rightarrow \mathbb{C}, \hat{\varphi} \mid \mathbb{A}\left(r_{0}, 1\right)=\varphi$. Next, the smooth Jordan curves $\mathcal{C}_{r}=\varphi(\mathbb{S}(r))$ are quasicircles. We seek optimal bounds on the distortion of these quasicircles.

3.1. Quasicircles and conformal maps of annuli. Here is the main result of this section.

Theorem 3. Let $\varphi: \mathbb{A}\left(r_{0}, 1\right) \rightarrow \mathbb{D}$ with $\varphi(\mathbb{S})=\mathbb{S}$ be a conformal mapping. Then for $r_{0}<r \leq 1$, the smooth Jordan curve $\mathcal{C}_{r}=\varphi(\mathbb{S}(r))$ is a K-quasicircle with

$$
K \leq K_{0}=\frac{1+\tan \left(\frac{\pi}{4} \frac{\log (r)}{\log \left(r_{0}\right)}\right)}{1-\tan \left(\frac{\pi}{4} \frac{\log (r)}{\log \left(r_{0}\right)}\right)} .
$$

In fact the map $\varphi \circ r \circ \varphi^{-1}: \mathbb{S} \rightarrow \varphi(\mathbb{S}(r))$ has a $K_{0}$-quasiconformal extension to $\hat{\mathbb{C}}$.

Proof. We assume $\varphi$ has been extended by reflection. For

$$
\frac{r_{0}}{r}<|\eta|<\frac{1}{r r_{0}}
$$

and $\zeta \in X=\varphi(\mathbb{S}(r))$ we define

$$
\Phi_{\eta}(\zeta)=\varphi\left(\eta \varphi^{-1}(\zeta)\right) .
$$

Certainly $\Phi_{1}(\zeta)=\zeta, \Phi_{\eta}: X \rightarrow \mathbb{C}$ is an injection and $\Phi_{1 / r}(X)=\mathbb{S}$. Next, for each $\zeta \in X$ the map $\eta \mapsto \Phi_{\eta}(\zeta)$ is holomorphic. Thus, we have a motion of $X$ which 
moves it to a round circle when $\eta=1 / r$ and is holomorphic but not parameterised by the unit disk. We fix that by considering the universal cover. Let $h: \mathbb{D} \rightarrow$ $\mathbb{A}\left(r_{0} / r, 1 /\left(r r_{0}\right)\right)$ be the holomorphic universal covering map with $h(0)=1$ and $h^{\prime}(0)>0$. Set $\Psi(\lambda, \zeta)=\Phi_{h(\lambda)}(\zeta)$. Then $\Psi$ is a holomorphic motion of $X$ and extends to a motion of $\hat{\mathbb{C}}$ which at time $\lambda$ is $\frac{1+|\lambda|}{1-|\lambda|}$-quasiconformal.

We now have to relate $|\lambda|$ and the number $1 / r$ via $h$. We note that $\log \frac{1+|\lambda|}{1-|\lambda|}=$ $\rho_{\mathbb{D}}(0, \lambda)$. The covering map $h$ is a local isometry of the hyperbolic metrics, but as we have set things up it is an isometry on the interval $[0,1]$. Hence

$$
\log \frac{1+|\lambda|}{1-|\lambda|}=\rho_{\mathbb{A}\left(r_{0} / r, 1 / r r_{0}\right)}(h(0),|h(\lambda)|)=\rho_{\mathbb{A}\left(r_{0} / r, 1 / r r_{0}\right)}(1,1 / r)=\rho_{\mathbb{A}\left(r_{0}, 1 / r_{0}\right)}(r, 1),
$$

and so $\mathcal{C}_{r}$ is a $K$-quasicircle, where $\log K=\rho_{\mathbb{A}\left(r_{0}, 1 / r_{0}\right)}(1, r)$. We need the value $\rho_{\mathbb{A}\left(r_{0}, 1 / r_{0}\right)}(1, r)$. The hyperbolic metric density (curvature equal to -1 ) of this annulus can be found in $[3, \S 12.2]$ as

$$
d(z)=\frac{\pi}{2 \log \left(1 / r_{0}\right)} \frac{1}{|z| \cos \left(\frac{\pi \log |z|}{2 \log \left(1 / r_{0}\right)}\right)} .
$$

Then

$$
\rho_{\mathbb{A}\left(r_{0}, 1 / r_{0}\right)}(1, r)=\frac{\pi}{2 \log \left(1 / r_{0}\right)} \int_{r}^{1} \frac{d t}{t \cos \left(\frac{\pi \log t}{2 \log \left(1 / r_{0}\right)}\right)}=\log \frac{1+\tan \left(\frac{\pi}{4} \frac{\log (r)}{\log \left(r_{0}\right)}\right)}{1-\tan \left(\frac{\pi}{4} \frac{\log (r)}{\log \left(r_{0}\right)}\right)} .
$$

We now recognise that $K=\exp \left(\rho_{\mathbb{A}\left(r_{0}, 1 / r_{0}\right)}(1, r)\right)$, and this completes the proof.

Corollary 2. Let $\varphi: \mathbb{A}\left(1, r_{0}\right) \rightarrow \mathbb{D}, \varphi(\mathbb{S})=\mathbb{S}$, be conformal and $0 \leq \alpha<1$. Then $\varphi\left(\mathbb{S}\left(0, r_{0}^{\alpha}\right)\right)$ is a $K$-quasicircle with

$$
K_{\alpha}=\frac{1+\tan \left(\frac{\pi}{4} \alpha\right)}{1-\tan \left(\frac{\pi}{4} \alpha\right)} .
$$

In fact the map $\varphi \circ r \circ \varphi^{-1}: \mathbb{S} \rightarrow \varphi\left(\mathbb{S}\left(r_{0}^{\alpha}\right)\right)$ has a $K_{\alpha}$-quasiconformal extension to $\hat{\mathbb{C}}$, and this estimate is essentially best possible.

What we mean by "essentially best possible" is explained in the next section. However, for fixed $\alpha$ we expect that a sharp bound will have $K_{\alpha} \rightarrow 1$ as $r_{0} \rightarrow 0$.

3.2. Lower bounds and sharpness. The problem in finding examples is in getting anything other than coarse estimates on the distortion of general quasicircles, so we turn to ellipses again. Let $r<1$ and $E$ be the region bounded by the ellipse $\{(r+1 / r) \cos (\theta)+i(r-1 / r) \sin (\theta): 0 \leq \theta \leq 2 \pi\}$. Define the conformal map $\varphi_{r}: \mathbb{D} \backslash \mathbb{D}(0, r) \rightarrow E \backslash[-(r+1 / r),(r+1 / r)]$ by

$$
\varphi_{r}(z)=\frac{z}{r}+\frac{r}{z}
$$

For $r<s<1$, the Jordan curve $\varphi_{r}(\mathbb{S}(s))$ is an ellipse which is a $K$-quasicircle

$$
K=\sqrt{\frac{s^{2}+r^{2}}{s^{2}-r^{2}}}
$$

and no better by Lemma 1. We want to note here that the boundary values do not have such a good extension. This surely follows from work on extremal quasiconformal mappings of Reich-Strebel et al., but we give a new proof based on our work with $L^{1}$-minimisers, [2]. 
Lemma 2. Let $L(z)=a z+b \bar{z},|a|>|b|$. Then

$$
\inf \{K: \text { there is a } K \text {-quasiconformal } F: \mathbb{D} \rightarrow \mathbb{C} \text { with } F|\mathbb{S}=L| \mathbb{S}\}=\frac{|a|+|b|}{|a|-|b|}
$$

The unique extremal mapping here is $L$.

Proof. The inverse of the harmonic extension of the boundary values of $L^{-1}$ (that is, $L)$ is the unique minimiser of $\iint_{\mathbb{D}} \mathbb{K} d z, \mathbb{K}=\frac{1}{2}(K+1 / K)$, over maps with the same boundary values as $L$; see [2]. Therefore for any such $F$,

$$
\|\mathbb{K}(z, F)\|_{\infty} \geq \frac{1}{\pi} \iint \mathbb{K}(z, F) d z \geq \frac{1}{\pi} \iint \mathbb{K}(z, L) d z=\|\mathbb{K}(z, L)\|_{\infty},
$$

and the middle inequality is strict unless $F=L$. The result follows as $K \mapsto$ $\frac{1}{2}(K+1 / K)$ is convex increasing for $K \geq 1$.

Returning to our example, certainly $\varphi(\mathbb{D})=\mathcal{E} \neq \mathbb{D}$, but as $r \rightarrow 0$ the Riemann map $\psi: \mathcal{E} \rightarrow \mathbb{D}$ converges with all derivatives to the identity. So for small $r$ we expect (8) to be close to a lower bound. We can get explicit estimates for all $r$ by observing that the linear map $g(z)=(r+1 / r) z+(r-1 / r) \bar{z}$ can be used to define a $K^{\prime}=\frac{1+r^{2}}{1-r^{2}}$-quasiconformal reflection $\Phi: \mathcal{E}=g(\mathbb{D}) \rightarrow \hat{\mathbb{C}} \backslash \mathcal{E}=g(\hat{\mathbb{C}} \backslash \overline{\mathbb{D}})$, $\Phi \mid \partial \mathcal{E}=$ identity, by

$$
\Phi(z)=g\left(\frac{g^{-1}(z)}{\left|g^{-1}(z)\right|^{2}}\right) .
$$

Then, again using the reflection in the circle,

$$
F(z)= \begin{cases}\psi(z) & z \in \mathcal{E} \\ \psi \circ \Phi(z)|\psi \circ \Phi(z)|^{-2}, & z \in \mathbb{C} \backslash \mathcal{E}\end{cases}
$$

defines a $K^{\prime}$-quasiconformal homeomorphism of $\mathbb{C}$. It follows that the conformal $\operatorname{map} \psi \circ \phi: \mathbb{D} \backslash \mathbb{D}(0, r) \rightarrow \mathbb{D}$ has $(\psi \circ \phi)(\mathbb{S})=\mathbb{S}$ and $(\psi \circ \phi)(\mathbb{S}(s))$ is at least a $\tilde{K}=K / K^{\prime}$-quasicircle, so

$$
\tilde{K} \geq \frac{1-r^{2}}{1+r^{2}} \sqrt{\frac{s^{2}+r^{2}}{s^{2}-r^{2}}}
$$

We put $s=r^{\alpha}, 0<\alpha<1$, and, with Corollary 2,

$$
\frac{1-r^{2}}{1+r^{2}} \sqrt{\frac{1+r^{2-2 \alpha}}{1-r^{2-2 \alpha}}} \leq K \leq \frac{1+\tan \left(\frac{\pi}{4} \alpha\right)}{1-\tan \left(\frac{\pi}{4} \alpha\right)} .
$$
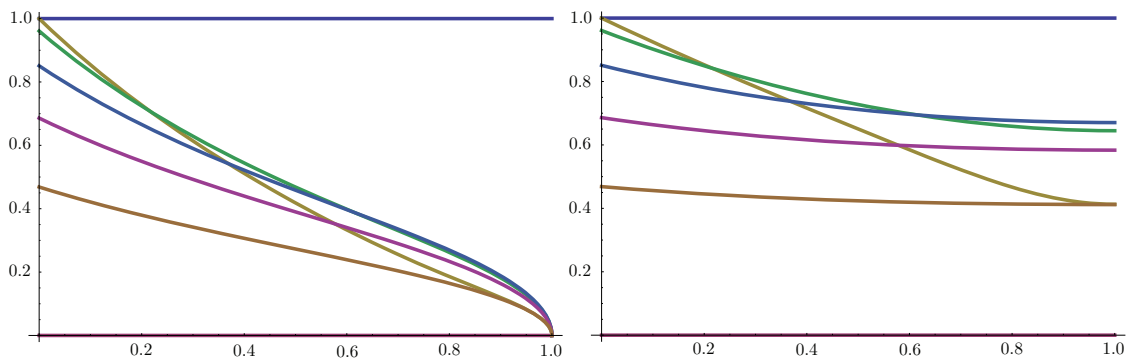

On the left is a graph of the left-hand side of (9) divided by the right-hand side of (9) for $r=0.01,0.2,0.4,0.6,0.8$ and $\alpha \in[0,1]$. In some sense the factor $\frac{1-r^{2}}{1+r^{2}}$ is artificial, but our result appears sub-optimal when $\alpha$ is close to 1 . However, as the 
linear boundary values do not admit such a good extension, the dilatation of the extension of the map $\varphi \circ r \circ \varphi^{-1}: \mathbb{S} \rightarrow \varphi\left(\mathbb{S}\left(r_{0}^{\alpha}\right)\right)$ of Corollary 2 is of the order of $\frac{1-r^{2}}{1+r^{2}} \frac{1+r^{2-2 \alpha}}{1-r^{2-2 \alpha}}$; there is no square-root. This is the graph on the right, showing that the distortion bounds on the extension are off by at most a constant factor and are "essentially sharp".

\section{Another applichtion}

Here we address the first question we raised concerning conformal maps of annuli. Unfortunately the result, though explicit, is not clean and is probably far from sharp.

Theorem 4. Let $\varphi: \mathbb{A}=\mathbb{A}(r, 1 / r) \rightarrow \mathbb{C}$ be conformal. Then $\varphi(\mathbb{S})$ is a $K$ quasicircle, and

$$
K \leq(1+\sqrt{2}) \frac{1+\sqrt{1-\left(\mu^{-1}(\log (1 / r))\right)^{2}}}{\mu^{-1}(\log (1 / r))},
$$

where $\mu(s)$ is the modulus of the Grötzsch ring $\mathbb{D} \backslash[0, s]$.

Proof. We can assume by an elementary limiting argument that $\varphi(\mathbb{S}(1 / r))$ is a Jordan curve bounding a domain $\Omega \subset \mathbb{C}$. Let $\psi: \Omega \rightarrow \mathbb{D}$ be the Riemann mapping. Accordingly, $(\psi \circ \varphi)(z / r)$ from $\mathbb{A}\left(r^{2}, 1\right) \rightarrow \mathbb{D}$ satisfies the hypotheses of Theorem 3 and $(\psi \circ \varphi) \mid \mathbb{S}(r)$ has a $\frac{1+\tan \left(\frac{\pi}{8}\right)}{1-\tan \left(\frac{\pi}{8}\right)}=(1+\sqrt{2})$-quasiconformal extension. Let $D=$ $\mathbb{D}(a, \rho)$ be the smallest hyperbolic disk in $\mathbb{D}$ containing $(\psi \circ \varphi)(\mathbb{S})$. Then $\psi^{-1} \mid D \rightarrow \mathbb{C}$ has a $\frac{e^{\rho}+1}{e^{\rho}-1}$-quasiconformal extension; see Corollary 2. Hence $\varphi(\mathbb{S})$ is a $K$-quasicircle for some $K \leq(1+\sqrt{2}) \frac{e^{\rho}+1}{e^{\rho}-1}$. We estimate $\rho$. The modulus of the annulus $\mathbb{A}(r, 1)$ is $\log (1 / r)$, which must be the modulus of the ring with boundary components $\mathbb{S}$ and $(\psi \circ \varphi)(\mathbb{S})$. Move $D$ via a Möbius transformation so the origin lies on its boundary. The extremal property of the Grötzsch ring implies $\log (1 / r) \leq \mu\left(\left(e^{2 \rho}-1\right) /\left(e^{2 \rho}+1\right)\right)$. As $\mu$ is increasing,

$$
\log \sqrt{\frac{1+\mu^{-1}(\log (1 / r))}{1-\mu^{-1}(\log (1 / r))}} \leq \rho,
$$

and the result follows.

As $\mu(s)=\frac{\pi}{2} \frac{\mathcal{K}\left(\sqrt{1-s^{2}}\right)}{\mathcal{K}(s)}, \mathcal{K}$ being the elliptic integral of the first kind, we can explore this bound computationally. However the results do not seem particularly compelling.

\section{REFERENCES}

[1] K. Astala, T. Iwaniec and G.J. Martin, Elliptic partial differential equations and quasiconformal mappings in the plane. Princeton Mathematical Series, 48. Princeton University Press, Princeton, NJ, 2009. MR2472875 (2010j:30040)

[2] K. Astala, T. Iwaniec, G.J. Martin and J. Onninen, Extremal mappings of finite distortion, Proc. London Math. Soc., 91 (2005), 655-702. MR2180459 (2006h:30016)

[3] A.F. Beardon and D. Minda, The hyperbolic metric and geometric function theory, Quasiconformal mappings and their applications, 9-56, Narosa, New Delhi, 2007. MR2492498 (2011c:30108)

[4] F.P. Gardiner, Y. Jiang and Z. Wang, Holomorphic motions and related topics, Geometry of Riemann surfaces, 156-193, London Math. Soc. Lecture Note Ser., 368, Cambridge Univ. Press, Cambridge, 2010. MR2665009 (2011j:37087) 
[5] Y. Jiang, Holomorphic motions, Fatou linearization, and quasiconformal rigidity for parabolic germs, Michigan Math. J., 58 (2009), 517-534. MR2595552(2011h:37075)

[6] S.L. Krushkal, Quasiconformal extensions and reflections, Handbook of complex analysis: geometric function theory, 2, 507-553, Elsevier, Amsterdam, 2005. MR2121866 (2006a:30018)

[7] R. Kühnau, Möglichst konforme Spiegelung an einer Jordankurve [Extremal conformal reflection in a Jordan curve], Jahresber. Deutsch. Math.-Verein., 90 (1988), 90-109. MR.939755 (89d:30023)

[8] R. Mané, P. Sad and D. Sullivan, On the dynamics of rational maps, Ann. Sci. École Norm. Sup., 16 (1983), 193-217. MR.732343 (85j:58089)

[9] G. J. Martin, The distortion theorem for quasiconformal mappings, Schottky's theorem and holomorphic motions, Proc. Amer. Math. Soc., 125 (1997), 1095-1103. MR.1363178 (97g:30017)

[10] G. J. Martin, Topology and distortion, preprint.

[11] D. Partyka, The maximal dilatation of Douady and Earle extension of a quasisymmetric automorphism of the unit circle, Ann. Univ. Mariae Curie-Sklodowska Sect. A, 44 (1990), 45-57 (1991). MR:1157878 (93h:30035)

[12] E. Reich and K. Strebel, Extremal plane quasiconformal mappings with given boundary values, Bull. Amer. Math. Soc., 79 (1973), 488-490. MR0338362 (49:3127)

[13] Z. Slodkowski, Holomorphic motions and polynomial hulls, Proc. Amer. Math. Soc., 111 (1991), 347-355. MR1037218 (91f:58078)

[14] Strebel, K., Extremal Quasiconformal Mappings, Results Math., 10 (1986), 168-210. MR869809(88a:30048)

Institute for Advanced Study, Massey University, Auckland, New Zealand

E-mail address: g.j.martin@massey.ac.nz 